\title{
Dielectric Breakdown Strength and Electrical Conductivity of Low Density Polyethylene Octylnanosilica Composite
}

\author{
S. Virtanen, A.S. Vaughan \\ ECS; University of Southampton \\ Southampton, United Kingdom \\ s.virtanen@soton.ac.uk \\ L.Yang, F. Saiz, N. Quirke \\ Department of Chemistry; Imperial College, \\ London, United Kingdom \\ n.quirke@ic.ac.uk
}

\begin{abstract}
One challenge in studying nanodielectric composites is to produce reliable, reproducible samples. A common strategy to suppress aggregation and make the particles more compatible with the polymer matrix is to modify the nanoparticle surface chemistry but, often, evaluation of the effectiveness of the chosen surface functionalization process can prove difficult. In this paper the emphasis is on feasible ways to monitor the production of silane coupled nanosilica low density polyethylene (LDPE) composites, using Fourier transform infrared spectroscopy (FTIR) and thermal gravimetric analysis (TGA). The AC-breakdown properties of the resulting composites is studied and the field dependency of the DCconductivity is measured and also calculated using a space charge limited conduction (SCLC) model together with densities of states obtained from ab initio calculations. For composites containing $13 \mathrm{wt} \%$ of nanosilica, breakdown strengths some $18 \%$ higher than that of the unfilled LDPE were obtained. However, the results are not stable over time. This appears to be related to how extensively the composite is dried at elevated temperatures under vacuum.
\end{abstract}

Keywords - polymer nanocomposite; nanosilica; nanodielectrics; solvent blending.

\section{INTRODUCTION}

Dielectric nanocomposites are polymeric materials that contain small amounts of inorganic particles which, to achieve good dispersion, have often modified surfaces to suppress aggregation and to make the particles more compatible with the polymer matrix [1]. However, if a solvent-based methodology is used to introduce the particles into the polymer matrix, there is a possibility that residual solvent will be retained in the system, for example, through preferential adsorption on nanoparticle surfaces. Indeed, the presence of even small quantities of polarizable moieties have been shown to affect electrical properties [2,3]. Also it has been shown that, in time, composites containing silica are capable of absorbing water, which can lead to a reduction in the breakdown strength. $[4,5]$.
In the work reported here, surface functionalized nanosilica/LDPE nanocomposites, which show enhanced electrical breakdown performance relative to unfilled polymers, were stored for four months and extensively dried and studied again, to see if the initial improvement were stable over time.

\section{EXPERIMENTAL}

\section{A. Materials and Processing}

Two different sets of nanocomposites are considered here, all of which were based upon low density polyethylene (LDPE). The LDPE was LD100BW (ExxonMobil) and the nanosilica was obtained from Sigma Aldrich with a quoted particle size of 10-20 nm. One set of nanocomposites was formulated using the nanosilica as-supplied, while the other contained octylsilane functionalized nanosilica. In order to modify the surface of nanosilica powder, a suspension of the nanosilica (10 g in $100 \mathrm{ml})$ in dry tetrahydrofuran (THF) was produced in a two-necked round-bottom flask and was flushed with dry nitrogen gas. Then, triethoxy(octyl)silane $(10 \mathrm{ml}$, $\sim 30 \mathrm{mmol}$ ) was added and the reaction mixture was refluxed for $8 \mathrm{~h}$ in a dry nitrogen atmosphere, before being cooled to room temperature. The reaction mixture was next poured into an excess of hexane $(500 \mathrm{ml})$ and the particles were allowed to precipitate. Thereafter, the functionalized nanosilica was redispersed in $20 \mathrm{ml}$ of acetone, re-precipitated in $200 \mathrm{ml}$ of hexane before, finally, being dispersed in a small amount of THF prior to use and characterization. Samples of both the untreated and functionalized nanosilicas were analyzed using Fourier transform infrared (FTIR) spectroscopy and thermogravimetric analysis (TGA) to determine the success of functionalization.

All nanocomposites were prepared using a solvent dispersion route. The matrix LDPE, which contained commercial thermal stabilizers, was dissolved in boiling 
xylene, within which the nanoparticles had already been dispersed. In addition, a reference sample (PEx) was also formulated in an identical way, but without the inclusion of any particles. After the polymer had dissolved, the solvent was removed in a rotary evaporator and the resulting product was dried under dynamic vacuum for $24 \mathrm{~h}$ at $60^{\circ} \mathrm{C}$. Sample sheets were pressed at $180^{\circ} \mathrm{C}$. A second reference system (PE) was also prepared by direct melt pressing of polymer pellets, to reveal the effect of solvent processing. The sample nomenclature is shown in Table 1.

After preparation, all samples were stored overnight in a desiccator and analyzed during the following day. For all nanocomposites, the filler loading level and removal of solvent was confirmed using TGA.

To monitor the long-term stability of the composites, samples were stored in a desiccator for four months, after which, they were thoroughly dried under dynamic vacuum at $80{ }^{\circ} \mathrm{C}$ until the weight of each sample remained constant. This took approximately one week.

\section{B. Electrical Measurements}

The DC-conductivity measurements were made using a stepped voltage technique. In this, the voltage was increased from $100 \mathrm{~V}$ to a value equivalent to an applied electric field of $40 \mathrm{kV} / \mathrm{mm}$ at $100 \mathrm{~V}$ steps; each voltage was held for $10 \mathrm{~s}$ before the current was recorded. The samples used were $\sim 0.2 \mathrm{~mm}$ in thickness and $30 \mathrm{~mm}$ in diameter, onto which, gold electrodes were sputter coated on each side.

AC-breakdown tests were conducted using a Phenix tester by applying a $50 \mathrm{~V} / \mathrm{s}$ ramp $\mathrm{AC}$ voltage to a thin specimen immersed in silicone oil. The samples were $0.10 \mathrm{~mm}$ to $0.13 \mathrm{~mm}$ in thickness, $6.25 \mathrm{~mm}$ diameter sphere electrodes were used and 20 breakdown measurements were made for each sample. The resulting breakdown data were analyzed assuming a 2-parameter Weibull distribution; the scale parameter, $\alpha$, was used to define the breakdown strength while the shape parameter $\beta$ provides a measure of the scatter in the data.

TABLE I. STUDIED MATERIALS

\begin{tabular}{|l|c|c|c|c|}
\hline \multicolumn{1}{|c|}{$\begin{array}{c}\text { Sample } \\
\text { Name }\end{array}$} & $\begin{array}{c}\text { Polymer } \\
\text { matrix }\end{array}$ & Filler & processing & $\begin{array}{c}\text { Filler } \\
\text { loading } \text { wt\% }\end{array}$ \\
\hline $\mathrm{PE}$ & LDPE & - & - & - \\
\hline $\mathrm{PEx}$ & $\mathrm{LDPE}$ & - & $\begin{array}{c}\text { xylene } \\
\text { blending }\end{array}$ & - \\
\hline $\mathrm{PEx} / \mathrm{Si} / 7$ & $\mathrm{LDPE}$ & silica & $\begin{array}{c}\text { xylene } \\
\text { blending }\end{array}$ & 7 \\
\hline $\mathrm{PEx} / \mathrm{C} 8 \mathrm{Si} / 13$ & $\mathrm{LDPE}$ & $\begin{array}{c}\text { octyl- } \\
\text { silica }\end{array}$ & $\begin{array}{c}\text { xylene } \\
\text { blending }\end{array}$ & 13 \\
\hline
\end{tabular}

\section{RESULTS AND DISCUSSION}

\section{A. Nanosilica Analysis}

Fig. 1 compares FTIR data obtained from (a) triethoxy(octyl)silane, (b) the as-supplied nanosilica and (c) the nanosilica after functionalization. From these data, the presence of octyl groups on surface of silica is confirmed by the characteristic peaks arrowed in Fig. 1. Fig. 2 shows the time derivative of the sample weight as a function of temperature for (a) unwashed functionalized nanosilica, (b) washed functionalized silica and (c) the as-supplied silica. These traces have been vertically offset for clarity of presentation. In trace (c), two degradation processes are evident at temperatures below $200^{\circ} \mathrm{C}$, which correspond to the loss of bound water and the THF in which all the studied particles were dispersed, in order to transfer them into a TGA pan for analysis. The range $400-600^{\circ} \mathrm{C}$ corresponds to the degradation of surface bound material and unattached silane residue; the latter is evinced by mass loss peak below $300^{\circ} \mathrm{C}$. The functionalization reduces adsorbed moisture, as the peak associated with surface bound water above $100^{\circ} \mathrm{C}$ is seen in bare silica but not in functionalized silica.

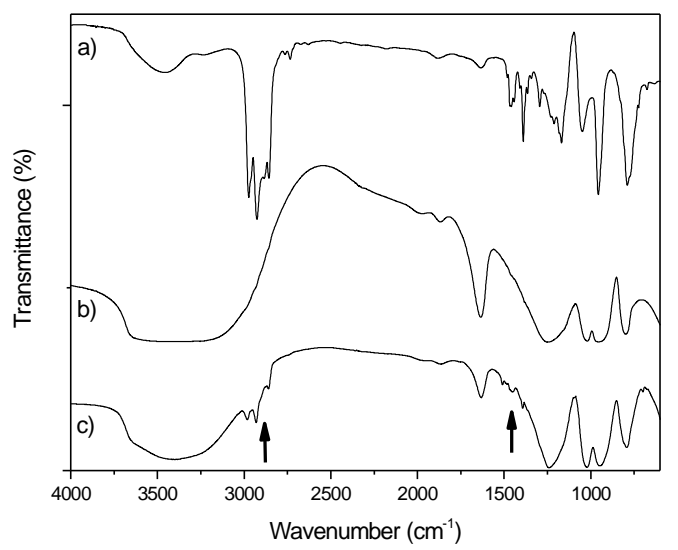

Fig. 1. FTIR spectra of a) triethoxy(octyl)silane b) silica c) triethoxy(octyl)silane functionalised silica. Arrows show peaks characteristic to triethoxy(octyl)silane.

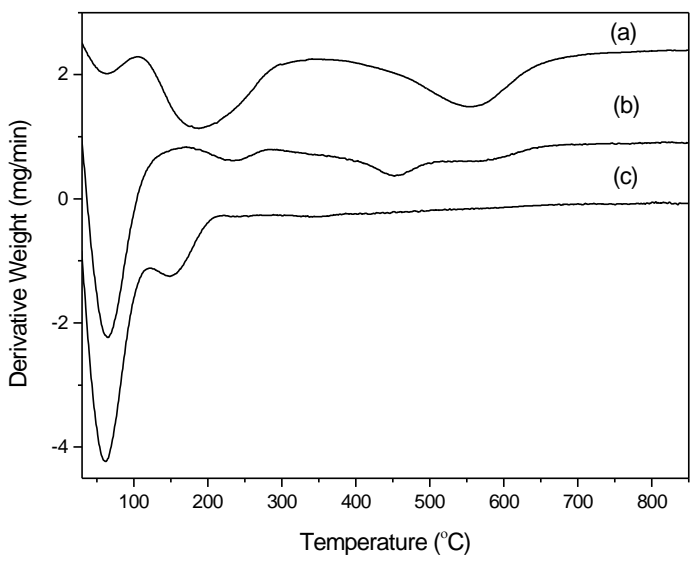

Fig. 2. The derivative weight as function of temperature for a) unwashed functionalised b) washed functionalised c) untreated silica nanoparticles. 


\section{B. Dielectric Breakdown}

Weibull plots of breakdown data obtained from selected samples are shown in Fig. 3, while the derived parameters are listed in Table 2. From this, it is evident that the solvent processed but unfilled PEx reference material and the $\mathrm{PEx} / \mathrm{C} 8 \mathrm{Si} / 13$ composite containing $13 \mathrm{wt} \%$ of octylfunctionalized nanosilica, both exhibit improved breakdown performance compared with the polyethylene that was meltpressed directly from pellets and the $\mathrm{PEx} / \mathrm{Si} / 7$ system, which contains $7 \mathrm{wt} \%$ of untreated nanosilica. Fig. 5 compares breakdown data obtained from $\mathrm{PEx} / \mathrm{C} 8 \mathrm{Si} / 13$ and $\mathrm{PEx} / \mathrm{Si} / 7$ as initially produced (indicated at start) and after four months storage and drying at $80^{\circ} \mathrm{C}$ in vacuum oven (indicated after drying). The vertical bars shown represent the Weibull scale parameter for the relevant system, normalized to the breakdown strength of PE: that is, the unfilled polymer that had not been subject to solvent processing. From Fig. 4, it can be seen that the breakdown strength of $\mathrm{PEx} / \mathrm{Si} / 7$ composite is largely invariant to drying, while the breakdown strength of PEx/C8Si/13 decreases by more than $20 \%$.

\section{Electrical Conduction}

The field-domain response of electrical conductivity measured contemporaneously with the breakdown strength, both initially and after drying, is shown in Fig. 5. The conductivities of the $\mathrm{PEx} / \mathrm{Si} / 7$ and $\mathrm{PEx} / \mathrm{C} 8 \mathrm{Si} / 13$ composites are similar at the start and much higher than the conductivities of the solvent blended PEx or the unprocessed PE samples. It can be seen that drying does not affect the unfilled samples (PE and PEx) but, after drying, the conductivities of both composites (PEx/Si/7 and $\mathrm{PEx} / \mathrm{C} 8 \mathrm{Si} / 13$ ) decrease to values that are comparable with those of the unfilled samples.

It is intriguing that both composites, $\mathrm{PEx} / \mathrm{Si} / 7$ and $\mathrm{PEx} / \mathrm{C} 8 \mathrm{Si} / 13$, show increased DC-conductivity but only the $\mathrm{PEx} / \mathrm{C} 8 \mathrm{Si} / 13$ system shows an increased AC-breakdown strength at the start. These materials also differ in their

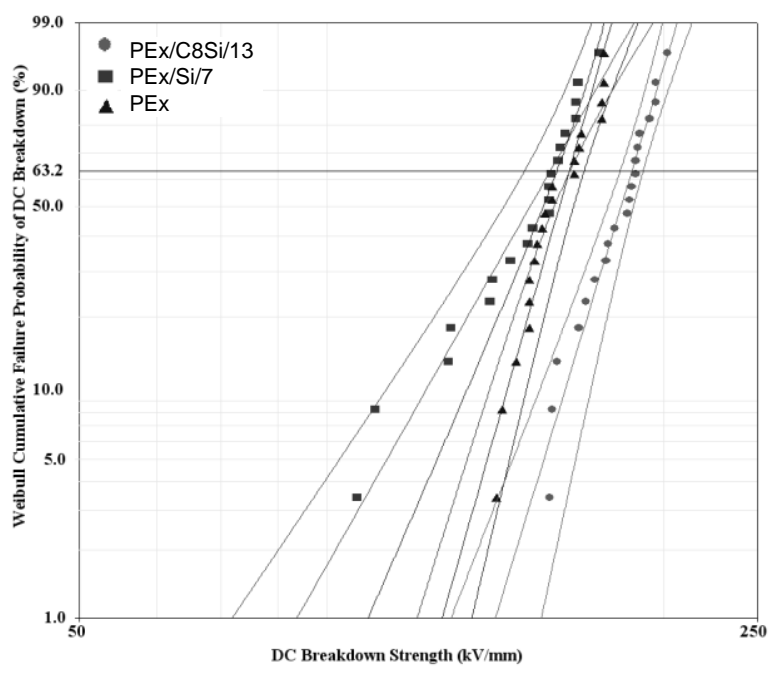

Fig. 3. Weibul plot of breadown data obtained from the indicated materials. The lines represent the calculated $90 \%$ confidence bounds
TABLE II. WEIBULL PARAMETERS FOR THE INDICATED SYSTEMS

\begin{tabular}{|l|c|c|c|}
\hline \multirow{2}{*}{ Sample } & \multirow{2}{*}{$\begin{array}{c}\text { Thickness } \\
(\mathbf{m m})\end{array}$} & \multicolumn{2}{|c|}{ Weibull parameters } \\
\cline { 3 - 4 } & & $\boldsymbol{\alpha}(\boldsymbol{k}$ V/mm $)$ & $\boldsymbol{\beta}$ \\
\hline $\mathrm{PE}$ & 0.13 & $152 \pm 4$ & 11 \\
\hline $\mathrm{PEx}$ & 0.11 & $160 \pm 5$ & 15 \\
\hline $\mathrm{PEx} / \mathrm{Si} / 7$ & 0.12 & $153 \pm 8$ & 8 \\
\hline $\mathrm{PEx} / \mathrm{C} 8 \mathrm{Si} / 13$ & 0.11 & $186 \pm 4$ & 14 \\
\hline $\mathrm{PE}$ dry & 0.10 & $144 \pm 5$ & 12 \\
\hline $\mathrm{PEx} / \mathrm{Si} / 7$ dry & 0.11 & $140 \pm 6$ & 9 \\
\hline $\mathrm{PEx} / \mathrm{C} 8 \mathrm{Si} / 13$ dry & 0.11 & $137 \pm 4$ & 12 \\
\hline
\end{tabular}

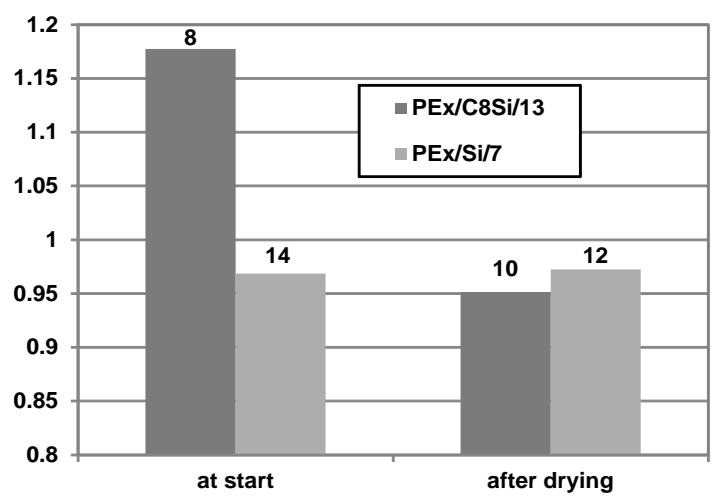

Fig. 4. The effect of vacuum drying on Weibull breakdown parameters for composites containing functionalized and unfuctionalized silica. The vertical bars represent $\alpha$ value normalised to PE sample; the numerical $\beta$ value for each data set is shown above each bar.

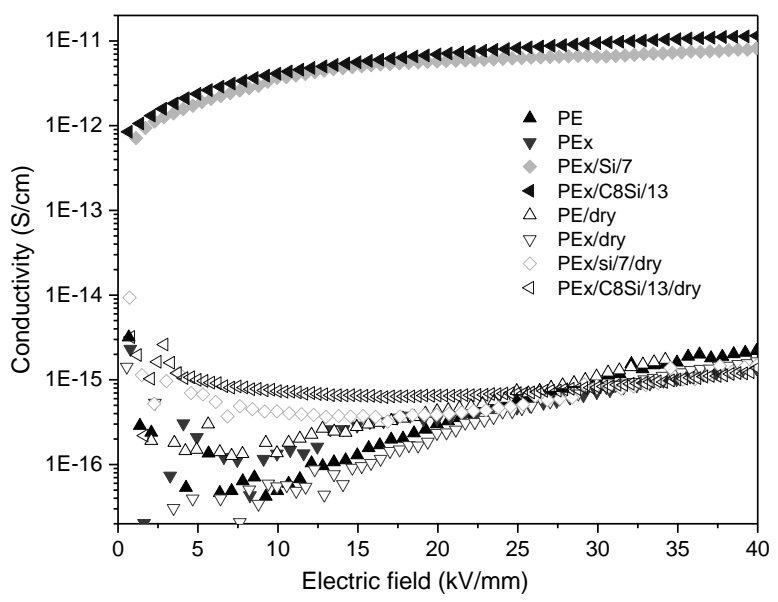

Fig. 5. DC-conductivity of different materials as a function of electric field when $10 \mathrm{~V} / \mathrm{s}$ ramp rate was used at $30{ }^{\circ} \mathrm{C}$

response to drying; this suggests that the silane treatment is the cause. Here, the composites were dried at a higher temperature than in previous studies [4, 5]. In these dryer samples the DC-conductivity is similar to the polymer matrix.

The pure polymer conductivity measurements can be understood using the SCLC model as implemented in Anta et al. [6]. The input to the model is the electron contact density, the dimensions of the sample and the density of excess 
electron states (DoS), which we divide into two contributions; the low energy (shallow) physical traps where the DoS is taken from $a b$ initio simulations of amorphous polyethylene [7] and the high energy (deep) chemical traps represented by a single trap [8]. The model solves a current-field equation together with the Poisson equation for the variation of charge density with field, in the absence of diffusion and polarization currents, and is based on a transport model involving electrons hopping from localized trap states to the mobility edge and then back down to a new localized state in a different position. Fig. 6 shows that the SCLC model provides a good description of the variation of conductivity with field with a reasonable value of the chemical trap depth; this is typical of the pure polymeric samples. The fit suggests that the low field experimental measurements are unreliable. In contrast, it was not possible to fit the nanocomposite data where it differs from the pure polymeric samples; Fig. 7 is a typical result. SCLC does not contain any representation of the nanoparticle component and it is clear that, at least for the as produced nanocomposites, additional contributions from the nanoparticles must be considered.

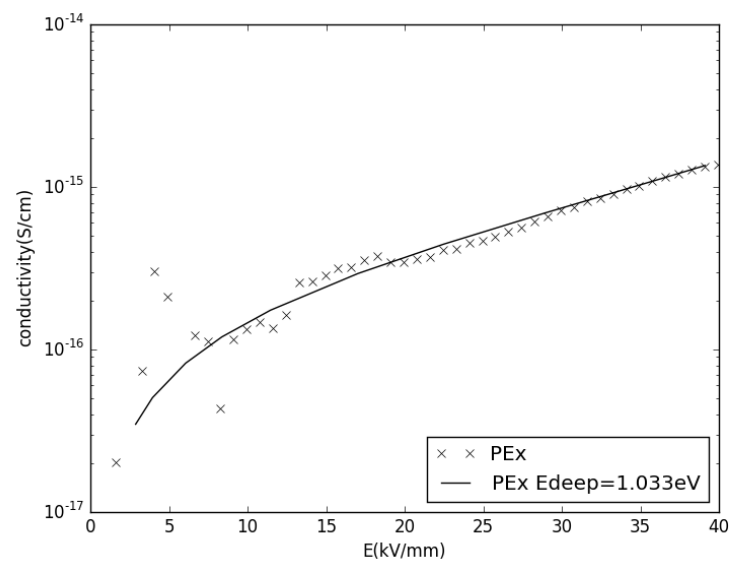

Fig. 6: SCLC model for xylene processed LDPE (PEx).

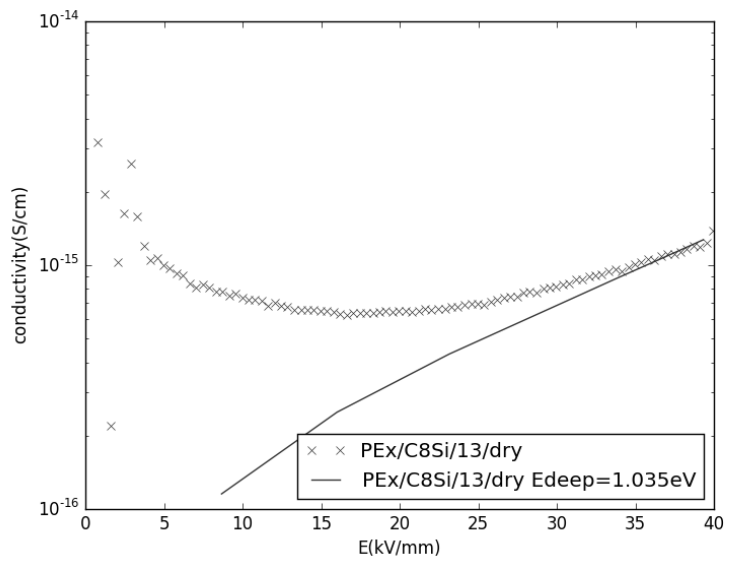

Fig. 7: SCLC model for xylene prosessed LDPE composite containing $13 \mathrm{wt} \%$ of octyl-functionalized nanosilica $(\mathrm{PEx} / \mathrm{C} 8 \mathrm{Si} / 13)$.

\section{CONCLUSIONS}

The octylsilane functionalized silica improved the electrical breakdown strength of LDPE. When the effect of vacuum drying was studied, it became apparent that the initial increase in AC-breakdown strength is not permanent. At the same time, there was a change in the DC-conductivity of the composite. More studies are needed to identify the underlying reasons for the initially observed increase in the breakdown strength of the composite, but it seems that it is related to the initial processing regime. The increase seen in breakdown strength may be related to the residual and loosely bound silanes, as the DCconductivity of the composite decreases after it is dried at elevated temperature.

This study points out that caution is required when evaluating the breakdown strength of composite materials. It is important to verify that the sample studied is a stable state and not, for example, prone to release any of its components or interact with its environment.

\section{ACKNOWLEDGMENT}

The authors would like to thank the Engineering and Physical Science Research Council EPSRC for supporting this work through the Advances Polymer material for Energy Security - POLYMAT grant, EP/N002288/1. All the data supporting this study are openly available from the University of Southampton repository

\section{REFERENCES}

[1] J.K. Nelson, Dielectric nanocomposites, Springer, 2010.

[2] M. Karlsson, "Investigation of the dielectric breakdown strength of polymer nanocomposites," 2014

[3] S. Virtanen T Krentz, L.S. Schadler J.K. Nelson, M. Bell, B. Benicewitz "Dielectric breakdown strength of epoxy bimodal polymer-brush-grafted core functionalized silica nanocomposites," IEEE Transactions on Dielectrics and Electrical Insulation, vol. 21, no. 2, pp. 563-570, 2014.

[4] K.Y. Lau, A.S.Vaughan, G. Chen, I.L. Hosier, A.F. Holt, "On the dielectric response of silica based polyethylene nanocomposites," J. Phys. D: Appl. Phys., Vol. 46, Art. No. 095303, 2013.

[5] I.L. Hosier, M, Praeger; A.F. Holt; A.S. Vaughan, S.G. Swingler, "Effect of water absorption on dielectric properties of nanosilica/polyethylene composites", Electrical Insulation and Dielectric Phenomena (CEIDP), IEEE Conference on, pp.651-654, 19-22 Oct. 2014.

[6] J.A. Anta, G. Marcelli, M. Meunier and N. Quirke, "Models of electron trapping and transport in polyethylene: Current-voltage characteristics," J. Appl. Phys., 92, pp. 1002-1008, 2002.

[7] Y. Wang, D. MacKernan, D. Cubero, D.F. Coker and N. Quirke, "Single electron states in polyethylene," J.Chem.Phys., 140, Art. No. 154902, 2014.

[8] for ab initio calculations of chemical trap depths see M. Meunier, N. Quirke and A. Aslanides, "Molecular modeling of electron traps in polymer insulators: Chemical defects and impurities," J. Chem. Phys., 115, pp. 2876-2881, 2001. 